\title{
SOME INFINITELY BASED VARIETIES OF GROUPS
}

\author{
Dedicated to the memory of Hanna Neumann
}

ROGER M. BRYANT

(Received 14 March 1972)

Communicated by M. F. Newman

Problem 11 of Hanna Neumann's book [3] asks whether the product variety $\mathfrak{B}_{4} \mathfrak{B}_{2}$ has a finite basis for its laws. (For any positive integer $k, \mathfrak{B}_{k}$ denotes the variety of all groups of exponent dividing $k$.) I think that $\mathfrak{B}_{4} \mathfrak{B}_{2}$ was being suggested as a plausible candidate for a variety without the finite basis property; of course, at a time when no such example was known. It is the primary object of this note to verify the fact that $\mathfrak{B}_{4} \mathfrak{B}_{2}$ is not finitely based. $\mathfrak{B}_{4} \mathfrak{B}_{2}$ provides, therefore, probably the simplest example known at present of a variety which is not finitely based.

Since the proof here is similar to that used by Vaughan-Lee [5], and even closer to that of Newman [4], it will be described in a somewhat abbreviated form.

For $n=1,2, \cdots$, let $v_{n}=x_{1}^{2} \cdots x_{n}^{2}$. Thus $\mathfrak{B}_{4} \mathfrak{B}_{2}$ has the basis $\left\{\left(v_{n}\right)^{4}: n=1,2, \cdots\right\}$ for its laws, and if it had a finite basis it would have a basis $\left\{\left(v_{n}\right)^{4}\right\}$ for some $n$. Consequently, it suffices to find, for each $n$, a group $G_{n}$ which satisfies $\left(v_{n}\right)^{4}$, but does not satisfy $\left(v_{m}\right)^{4}$ for some $m>n$.

Let $\mathfrak{I}_{2}$ be the variety generated by the dihedral group of order 8 , and let $A$ be a relatively free group of $\mathfrak{I}_{2}$ on some countable generating set. Write $U=A^{2}$ for the subgroup generated by the squares of elements of $A$. Let $B$ be a relatively free group of $\mathfrak{I}_{2}$ on a generating set $\{\beta(a): a \in A\}$ indexed by the elements of $A$. Write $V=B^{2}$. Thus $V$ is central in $B$ and has a free generating set (as abelian group of exponent 2)

$$
\left\{(\beta(a))^{2}: a \in A\right\} \cup\left\{\left[\beta\left(a_{1}\right), \beta\left(a_{2}\right)\right]: a_{1}, a_{2} \in A, a_{1} \neq a_{2}\right\} .
$$

Let $G$ be the semi-direct product of $B$ by $A$ in which $A$ and $B$ are regarded as subgroups of $G$ and

$$
\beta\left(a_{1}\right)^{a_{2}}=\beta\left(a_{1} a_{2}\right), \text { all } a_{1}, a_{2} \in A .
$$

A simple counting argument (see [4]) shows that there is an element $d$ of $U$ 
which is not a value of $v_{n}$ in $A$. But clearly, for some $m>n$, there are elements $c_{1}, \cdots, c_{m}$ of $A \backslash U$ such that $d=c_{1}^{2} \cdots c_{m}^{2}$. Write $c=c_{1}^{-1}$ and choose a set $T$ of coset representatives for $U$ in $A$ which contains both 1 and $c$. Let $R$ be the set of elements of $V$ of the form

$$
[\beta(t), \beta(t d)], t \in T,
$$

and let $S$ be the set of elements of $V$ of the forms

$$
(\beta(a))^{2}, a \in A,
$$

Take $J=\operatorname{gp}(R)$ and $K=\mathrm{gp}(S)$. It is easily seen that $R \cap S$ is empty, and that the distinct elements of $R \cup S$ form a basis for $V$. Hence $V=J \times K$. Also, $K$ contains all elements

$$
\left[\beta\left(a_{1}\right), \beta\left(a_{2}\right)\right]\left[\beta\left(a_{1} u\right), \beta\left(a_{2} u\right)\right], a_{1}, a_{2} \in A, u \in U,
$$

because any such element is a product of elements of types (iii) and (iv). The image under the action of an element of $A$ of an element of type (ii), (iii) or (iv) has type (ii), (iii) or (v), respectively. Since the elements of $S$ are central in $B$, it follows that $K$ is normal in $G$. We show that we can take $G_{n}=G / K$ in the above.

Every value of $v_{n}$ in $G$ has the form $u \beta\left(a_{1}\right) \cdots \beta\left(a_{k}\right)$, where $u \in U \backslash\{d\}$ and $a_{1}, \cdots, a_{k} \in A$. Now

$$
\left(u \beta\left(a_{1}\right) \cdots \beta\left(a_{k}\right)\right)^{4}=\left(\beta\left(a_{1} u\right) \cdots \beta\left(a_{k} u\right) \beta\left(a_{1}\right) \cdots \beta\left(a_{k}\right)\right)^{2},
$$

and this expands to

$$
\begin{aligned}
& \prod_{i}\left(\beta\left(a_{i} u\right)\right)^{2}\left(\beta\left(a_{i}\right)\right)^{2} \times \prod_{i}\left[\beta\left(a_{i}\right), \beta\left(a_{i} u\right)\right] \\
& \times \prod_{i>j}\left[\beta\left(a_{i} u\right), \beta\left(a_{j} u\right)\right]\left[\beta\left(a_{i}\right), \beta\left(a_{j}\right)\right] \times \prod_{i>j}\left[\beta\left(a_{i}\right), \beta\left(a_{j} u\right)\right]\left[\beta\left(a_{j}\right), \beta\left(a_{i} u\right)\right],
\end{aligned}
$$

which is a product of elements of types (ii), (iii) and (v). It follows that every value of $\left(v_{n}\right)^{4}$ in $G$ lies in $K$, and so $\left(v_{n}\right)^{4}$ is a law of $G / K$.

Clearly,

$$
\left(\beta(1) c_{1}\right)^{2} c_{2}^{2} \cdots c_{m}^{2}=\beta(1) \beta(c) c_{1}^{2} c_{2}^{2} \cdots c_{m}^{2}=\beta(1) \beta(c) d
$$

is a value of $v_{m}$ in $G$. Now

$$
(\beta(1) \beta(c) d)^{4}=(\beta(1) \beta(c) \beta(d) \beta(c d))^{2},
$$

and this expands to a product of elements of types (ii) and (iii) together with a term

$$
[\beta(1), \beta(d)][\beta(c), \beta(c d)],
$$


which is a non-trivial element of $J$. It follows that $\left(v_{m}\right)^{4}$ has a value in $G$ which lies outside $K$, and so $\left(v_{m}\right)^{4}$ is not a law of $G / K$. This completes the proof of the fact that $\mathfrak{B}_{4} \mathfrak{B}_{2}$ is not finitely based.

We show now how the proof above can be modified to give rather more, which we state as a theorem. This, again, could easily be extended further, but it is sufficient to yield some applications we wish to make. Let $\left[\mathfrak{B}_{2} \mathfrak{I}_{2}, \mathfrak{B}_{2}\right]$ denote the variety of those groups in which the $\mathfrak{B}_{2} \mathfrak{I}_{2}$ verbal subgroup is central in the $\mathfrak{B}_{2}$ verbal subgroup. Our result is

THEOREM. Let $k$ be an odd positive integer and $\mathfrak{B}$ any variety satisfying

$$
\mathfrak{B}_{4} \mathfrak{B}_{2} \wedge\left[\mathfrak{B}_{2} \mathfrak{I}_{2}, \mathfrak{B}_{2}\right] \subseteq \mathfrak{W} \subseteq \mathfrak{B}_{4} \mathfrak{B}_{2 k} .
$$

Then $\mathfrak{B}$ is not finitely based.

The variety $\mathfrak{B}_{4} \mathfrak{B}_{2} \wedge\left[\mathfrak{B}_{2} \mathfrak{I}_{2}, \mathfrak{B}_{2}\right]$ seems "smaller" than the other known examples of non-finitely-based varieties. It consists of groups of exponent dividing 8 and is contained in both $\mathfrak{I}_{2} \mathfrak{I}_{2}$ and $\mathfrak{C B}_{2}$, where $\mathfrak{C}$ denotes the variety of all centre-by-metabelian groups. The theorem has as a special case the fact that $\mathfrak{B}_{4} \mathfrak{B}_{2}$ is not finitely based, and also that $\left(\mathfrak{C} \wedge \mathfrak{B}_{4}\right) \mathfrak{B}_{2}$ is no: finitely based. Therefore, $\mathfrak{B}_{4}$ and $\mathfrak{C} \wedge \mathfrak{B}_{1}$, both of which are finitely based, are not strongly finitely based in the sense of [1]. One example of this sort has already been given by VaughanLee [6].

Let $p$ be any odd prime. Newman [4] gives a finitely based variety $\mathfrak{D}$, of soluble groups of exponent dividing $p^{3}$, having a subvariety which is not finitely based. (In fact, $\mathfrak{D}=\left(\mathfrak{A}_{p} \mathfrak{H}_{p} \wedge \mathfrak{N}_{p}\right) \mathfrak{I}_{p}$.) It follows, by a result of Kovács [2], that $\mathfrak{D}$ has $2^{\aleph_{0}}$ subvarieties. Thus the set of varieties $\mathfrak{M}$ satisfying

$$
\left(\mathfrak{C} \wedge \mathfrak{B}_{4}\right) \mathfrak{B}_{2} \subseteq \mathfrak{B} \subseteq\left(\mathfrak{C} \wedge \mathfrak{B}_{4}\right) \mathfrak{B}_{2} \mathfrak{D}
$$

has cardinality $2^{\aleph_{0}}$. Taking $k=p^{3}$ in the theorem, we find that this set consists entirely of varieties which are not finitely based. Consequently, the set of finitely based varieties is far from dense in the lattice of varieties of groups.

To prove the theorem, note first that, since $k$ is odd,

$$
\mathfrak{B}_{4} \mathfrak{B}_{2} \wedge\left[\mathfrak{B}_{2} \mathfrak{I}_{2}, \mathfrak{B}_{2}\right]=\mathfrak{B}_{4} \mathfrak{B}_{2 k} \wedge\left[\mathfrak{B}_{2} \mathfrak{I}_{2}, \mathfrak{B}_{2}\right] \wedge \mathfrak{B}_{16} \text {. }
$$

Therefore, by the modular law,

$$
\mathfrak{W}=\mathfrak{B}_{4} \mathfrak{B}_{2 k} \wedge\left(\mathfrak{B} \vee\left(\left[\mathfrak{B}_{2} \mathfrak{I}_{2}, \mathfrak{B}_{2}\right] \wedge \mathfrak{B}_{16}\right)\right) .
$$

For $n=1,2, \cdots$, let $u_{n}=x_{1}^{2 k} \cdots x_{n}^{2 k}$, and let $\mathfrak{w}$ be any basis for

$$
\mathfrak{B} \vee\left(\left[\mathfrak{B}_{2} \mathfrak{I}_{2}, \mathfrak{B}_{2}\right] \wedge \mathfrak{B}_{16}\right) \text {. }
$$

Thus $\mathfrak{M}$ has the basis $\mathfrak{w} \cup\left\{\left(u_{n}\right)^{4}: n=1,2, \cdots\right\}$, and if it had a finite basis it would have a basis $\mathfrak{w} \cup\left\{\left(u_{n}\right)^{4}\right\}$ for some $n$. Now, it may be verified that the groups $G_{n}$ 
constructed above lie in both $\left[\mathfrak{B}_{2} \mathfrak{I}_{2}, \mathfrak{B}_{2}\right]$ and $\mathfrak{B}_{16}$. Since $G_{n}$ satisfies $\left(v_{n}\right)^{4}$ and $k$ is odd, $G_{n}$ satisfies $\left(u_{n}\right)^{4}$; and, since $G_{n}$ does not satisfy $\left(v_{m}\right)^{4}$ for some $m>n, G_{n}$ does not satisfy $\left(u_{m}\right)^{4}$ for some $m>n$. Thus, for each $n, G_{n}$ satisfies $\mathfrak{w} \cup\left\{\left(u_{n}\right)^{4}\right\}$, but not $\left(u_{m}\right)^{4}$ for some $m>n$. It follows that $\mathfrak{B}$ is not finitely based.

\section{Acknowledgement}

The author is grateful to Dr. L. G. Kovács and Dr. M. F. Newman for several useful comments.

\section{References}

[1] M. S. Brooks, L. G. Kovács, and M. F. Newman, 'A finite basis theorem for product varieties of groups', Bull. Austral. Math. Soc. 2 (1970), 39-44.

[2] L. G. Kovács, 'On the number of varieties of groups', J. Austral. Math. Soc. 8 (1968), 444-446.

[3] Hanna Neumann, Varieties of Groups (Springer-Verlag, Berlin, 1967).

[4] M. F. Newman, 'Just non-finitely-based varieties of groups', Bull. Austral. Math. Soc. 4 (1971), 343-348.

[5] M. R. Vaughan-Lee, 'Uncountably many varieties of groups', Bull. London. Math. Soc. 2 (1970), 280-286.

[6] M. R. Vaughan-Lee, 'On product varieties of groups', Bull. Austral. Math. Soc. 5 (1971), 239-240.

Australian National University

Canberra, ACT, 2600

Present address

Institute of Science and Technology

University of Manchester

England 